\title{
Three new species of the Phaonia acerba group (Diptera: Muscidae) from Palaearctic and Oriental Regions
}

\author{
Teng Yu \& Qi W. Xue
}

Yu, T. \& Xue, Q. W. 2015: Three new species of the Phaonia acerba group (Diptera: Muscidae) from Palaearctic and Oriental Regions. - Entomol. Fennica 26: $1-7$.

Three new species of the Phaonia acerba group from the Palaearctic and Oriental Regions are described: P. gaoligongshanensis Xue \& Yu, sp. n., P. zhangxianggi Xue \& Yu, sp. n. and $P$. hongkuii Xue \& Yu, sp. n. An identification key to the males of 8 species of the Phaonia acerba group from the Palaearctic and Oriental Regions is provided.

T. Yu, Institute of Entomology, Shenyang Normal University, 110034, Shenyang, China; E-mail:772962099@qq.com

Q. W. Xue, Institute of Entomology, Shenyang Normal University, 110034, Shenyang, China; E-mail: xwqfly@sina.com

Received 27 October 2013, accepted 9 July 2014

\section{Introduction}

Phaonia was established by Robineau-Desvoidy (1830), with $P$. viarum as the type species. It is one of the largest genera in the family of Muscidae, with more than 800 species all over the world. The genus and species from the Palaearctic Region were first divided into 16 groups (Ringdahl 1949), then 18 groups (Hennig 1963) and later 21 groups and three subgroups (Zinovjev 1981). Following the work of these earlier researchers, Chinese scholars have concentrated on the study of Phaonia by recording 121 species in the key to the common flies of China (Fan 1992), 189 species in the flies of China (Xue 1996), and 312 species, including 68 new species, 38 groups and 18 subgroups in the Fauna Sinica Insecta (Ma et al. 2002). Phaonia acerba group is distinguished by the bare basisternum of prosternum, male frons distinctly narrower than in females, a majority of males without orbital seta, abdomen without yellow areas, mid tibia with 2 rows of posterior setae, epistoma situated front of frontal angle in profile, legs with yellow areas, and katepimeron sometimes bare.

The species of the group are mainly distributed in Palaearctic-Oriental Regions, but they are not found in Japan (Shinonaga 2003) at present.

\section{Material and methods}

The specimens examined for this paper were collected by sweeping brushwood in mountainous regions of the Southeast and Central China, using entomological nets. Genital structures were detached from the body, cleared by warming in a $10 \% \mathrm{NaOH}$ solution (approximately $120^{\circ} \mathrm{C}$ ) for fifteen minutes, placed in a droplet of glycerol, and observed under a compound light microscope.

The type specimens are deposited in the Institute of Entomology, Shenyang Normal University, Shenyang, China.

The morphological terminology follows that 
of McAlpine (1981). Measurements of the body length are in millimeters ( $\mathrm{mm})$. The following abbreviations are used for characters: $f r$, frontal setae; $a c r$, acrostichal setae; $d c$, dorsocentral setae; post $d c$, postsutural dorsocentral setae; ial, intra-alar setae; pra, prealar setae; $a v$, anteroventral setae; $a d$, anterodorsal setae; $p d$, posterodorsal setae; $p$, posterior setae; $p v$, posteroventral setae; $\mathrm{R}_{4+5}$, branch of radius; $\mathrm{M}$, medial vein.

\section{Descriptions of new species}

\subsection{Phaonia gaoligongshanensis Xue \& Yu, sp. n. (Fig. 1)}

Type material. Holotype. Male, China: Mount Gaoligong (type locality), Yunnan Province $\left(28.25^{\circ} \mathrm{N}, 98.14^{\circ} \mathrm{E}\right.$; altitude $\left.2,950 \mathrm{~m}\right), 6 . \mathrm{V} .2001$, Feng Y. Tong leg. Paratypes. 10 males, same data as holotype.

Description. Male. Body length 7.0-7.3 mm. Head. Eye covered with long lightish ciliae, frons narrower than anterior ocellus width, fronto-orbital plate adjoining, 7 to 8 pairs of $f r$ on lower half of frons, a pair of orbital setae shorter than eye ciliae length in front of anterior ocellus, fronto-orbital plate and parafacial with grey yellow pruinosity; parafacial subequal in width to antenna basal diameter; antenna black, postpedicel 3.0 times as long as wide, arista long plumose, the longest hair about twice as long as antenna basal diameter, epistoma situated front of anterior margin of frontal angle in profile, posterior margin of gena with 2 rows of subvibrissal setulae, gena about one fifth of eye in height, dorsal area of occiput with hairs, genal and postgenal hairs all black; prementum covered with pruinosity, 3.0 times as long as high, palpus yellow on basal half, dark brown in distal half.

Thorax. Entirely black in ground color, slightly light, sparsely covered with grey pruinosity, scutum with 4 black vittae; acr $0+1, d c 2+4$,

Fig. 1. Phaonia gaoligongshanensis Xue \& Yu, sp. n., male. - a. Sternite 5 in ventral view. - b. Cerci in posterior view. $-c$. Cerci and surstylus in profile. Scale bar $=0.5 \mathrm{~mm}$.
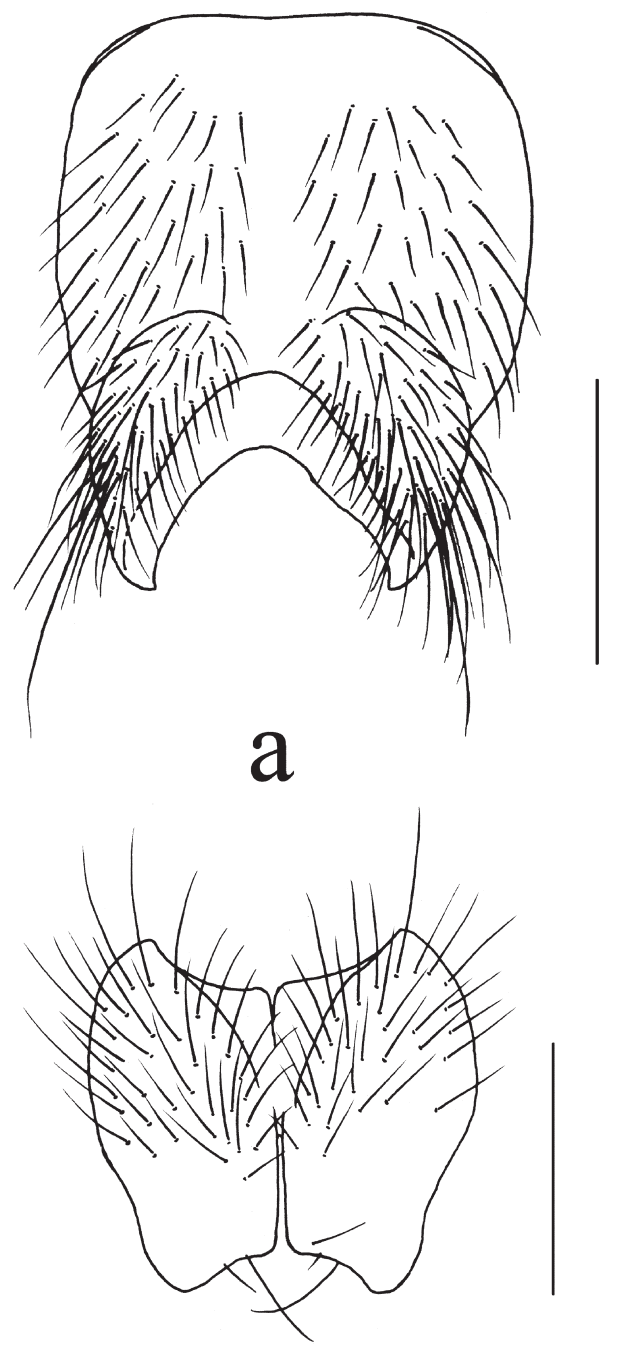

b

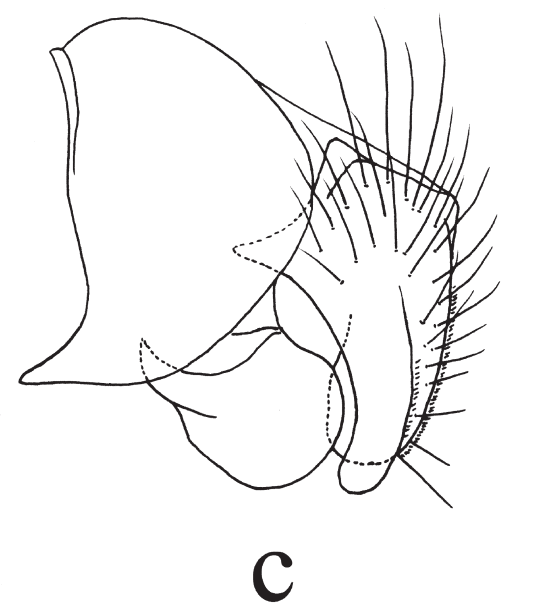


ial $0+2$, pra about twice as long as posterior notopleural setae, notopleuron with hairs, lateral and ventral scutellum bare, basisternum of prosternum, anepimeron, meron and katepimeron all bare, anterior spiracle brownish, posterior spiracle brown, katepisternum setae $1+2$.

Wings. Hyaline, veins brown, basicosta yellow, without costal spine, veins bare, except for C with hairs, $\mathrm{R}_{4+5}$ and $\mathrm{M}$ straight; calypter and haltere yellow.

Legs. Coxae, trochanter, femora and tibiae yellow, tarsi black; fore tibia av 2; mid femur $p v 3$ to 4 distinct on basal half, apical $a 1$, distal $p d 3$, mid tibia $p 4, p v 3$; hind femur without $p v$, ad becoming distinct apically, hind tibia $a v 5$, ad row irregular, including 2 setae strong, $p d 1$, without apical $p v$; tarsi longer than tibiae, claws and pulvilli small.

Abdomen. Black in ground color, round in dorsal view, sparsely covered with light grey pruinosity, without distinct vitta and shining patch; sternite 1 bare, outer process longer than inner process.

Female. Unknown.

Etymology. The species name is derived from the locality.

Remarks. This new species belongs to Phaonia acerba group. It resembles $P$. kangdingensis Ma \& Feng, 1985, but differs from it in the male characters: $f r$ in the lower half of frons ( $f r$ complete in $P$. kangdingensis), gena one fifth of eye in height (gena one fourth of eye in height in $P$. kangdingensis), palpus balck except yellow on basal half, dark brown on distal half (palpus entirely black in P. kangdingensis), basicosta yellow (basicosta black in $P$. kangdingensis), front of fore tibia yellow (front of fore tibia black in $P$. kangdingensis), mid tibia with $4 p, 3 p v$ (mid tibia with $3 p, 2 p v$ in $P$. kangdingensis), abdomen without distinct vitta (tergites with medium black vitta in $P$. kangdingensis), distal third of cercus narrowed (distal third of circus wide in $P$. kangdingensis).

\subsection{Phaonia hongkuii Xue \& Yu, sp. n.} (Fig. 2)

Type material. Holotype. Male, China: Moirigkawagarbo (type locality), Yunnan Province, $\left(27.02^{\circ} \mathrm{N}, 99.26^{\circ} \mathrm{E}\right.$; altitude 2,900 m), 31.V.2013, Kui H. Sun leg. Paratype. 1 male, same data as holotype.

Description. Male. Body length 7.4-7.6 mm. Head. Eye sparsely covered with short brownish ciliae, the longest ciliae about 1.5 times as long as anterior ocellus width, frons about 1.5 times as wide as anterior ocellus width, the narrowest area of fronto vitta about half to two thirds of frontoorbital plate in width, fr 9 to 11 pairs, upper half 4 to 5 pairs short and small, subequal in length to the longest eye ciliae, without orbital seta, frontoorbital plate and parafacial with light grey pruinosity; parafacial without a dark patch at the level of pedicel, subequal in width to antenna basal diameter; antenna black, postpedicel 3 times as long as wide, arista long plumose, the longest hair about 1.5 times as long as antenna basal diameter, lunule dark-brownish, epistoma situated front of frontal angle in profile, margin of gena with 2 rows of subvibrissal setula, gena one third of eye in height with dark grey pruinosity, occiput dorsal area with hairs, gena and postgena hairs black entirely; prementum covered with pruinosity, 4.0 times as long as high, palpus black and slender, 1.3 times as long as prementum length.

Thorax. Black in ground color, slightly light, sparsely covered with grey pruinosity, scutum with 4 black vittae; inner vittae on scutum not reaching to scutoscutellar suture, acr $0+1, d c$ $2+4$, ial $0+2$, pra about twice as long as posterior notopleural seta length, notopleuron with hairs, lateral and ventral scutellum bare, basisternum of prosternum, anepimeron, meron and katepimeron all bare, anterior spiracle orange to yellow, posterior spiracle brown, katepisternum setae $1+2$.

Wings. Hyaline, basal part of wing (including veins) yellow, other areas brown, basicosta yellow, costal spine short and small, subequal in length to $\mathrm{C}$ diameter, all veins bare, except for $\mathrm{C}$ with hairs, $\mathrm{R}_{4+5}$ and $\mathrm{M}$ straight; calypter and haltere brownish.

Legs. Coxae, trochanter, femora and tibiae yellow, basal of fore tibia black, tarsi black; fore tibia $a v 2$, mid femur without distinct $a v$, sub-apical $a 1,3$ to $4 p v$ on basal half, apical $p d 3$, mid tibia $p 3, p v 2$; hind femur with a row of $a v$, longer and stronger apically, without $p v$, hind tibia $a v 3$, 


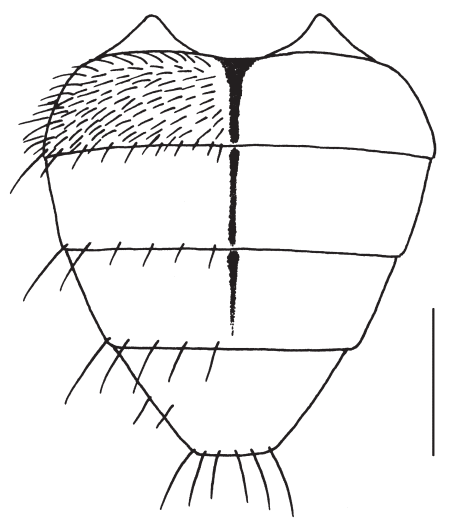

a

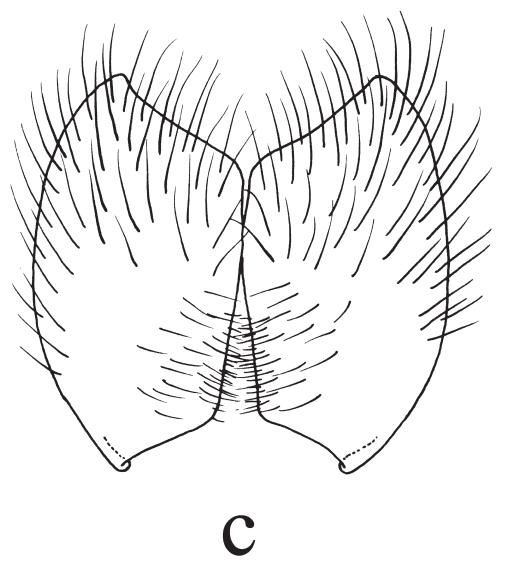

ad 2, pd 1 (absent on sub-basal), without apical $p v$; all tarsi longer than tibiae, fore leg claw longer than pulvillus, claw subequal in length to fifth tarsomere, hind legs claw and pulvilli short and small, subequal in length to two thirds of fifth tarsomere.

Abdomen. Black in ground color, triangled in dorsal view, sparsely covered with grey pruinosity in profile, as genus Ophyra; tergites 2 to 4 with a medium narrow black vitta, body hairs and setae short, sparse posteriorly, tergite 3 with 7 to 8 rows of body hairs; sternite 1 bare, sternite 2 to 4 nearly square, a pair of strong setae in distal margin, inner margin of cerci with hairs in dorsal view.

Female. Unknown.

Etymology. The species is named after the collector.

Remarks. This new species belongs to Phaonia acerba group. It resembles P. gaoli-
Fig. 2. Phaonia hongkuii Xue \& Yu, sp. n., male. - a. Abdomen in dorsal view, - b. Sternite 5 in ventral view. -c. Cerci in posterior view. - d. Cerci and surstylus in profile. Scale bars: $\mathrm{a}=1.0 \mathrm{~mm}$, $\mathrm{b}=0.5 \mathrm{~mm}, \mathrm{c}=0.2$ $\mathrm{mm}, \mathrm{d}=0.2 \mathrm{~mm}$. gongshanensis Xue \& Yu, sp. n., but differs from it in the male characters as shown in the key below. In addition, in Phaonia hongkuii Xue \& Yu, sp. n.: eye covered with short ciliae (eye covered with long ciliae in P. gaoligongshanensis), gena one third of eye in height (gena one fifth of eye in height in P. gaoligongshanensis), palpus entirely black (palpus black except yellow on basal half, dark brown on distal half in $P$. gaoligongshanensis), prementum 4.0 times as long as high (prementum 3.0 times as long as high in $P$. gaoligongshanensis), mid tibia without $p v$ (mid tibia with $3 p v$ in $P$. gaoligongshanensis), hind tibia with $5 a v$ (hind tibia with $3 a v$ in P. gaoligongshanensis). Phaonia hongkuii Xue \& Yu, sp. n., resembles also $P$. zhangxianggi Xue \& Yu, sp. n., but differs from it in the male characters as shown in the key below. In addition, in $P$. hongkuii Xue \& Yu, sp. n.: margin of inner free part of male cerci with distinct short hairs in pos- 
Fig. 3. Phaonia zhangxianggi Xue \& Yu, sp.

n., male - a. Abdomen in dorsal view. $-b$.

Sternite 5 in ventral view. - c. Cerci in posterior view. - d. Cerci and surstylus in profile. Scale bars: $\mathrm{a}=1.0 \mathrm{~mm}$, $\mathrm{b}=0.5 \mathrm{~mm}, \mathrm{c}=0.2$

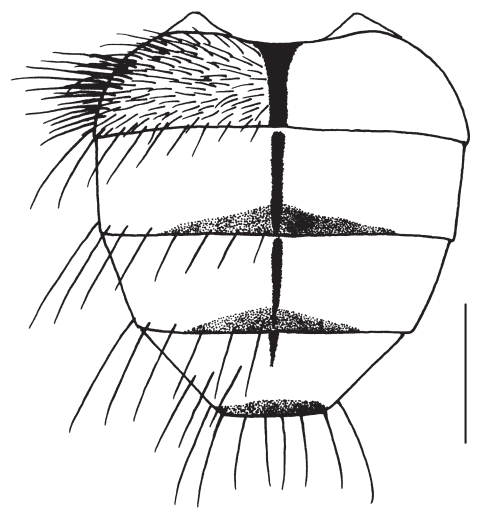

a $\mathrm{mm}, \mathrm{d}=0.2 \mathrm{~mm}$.
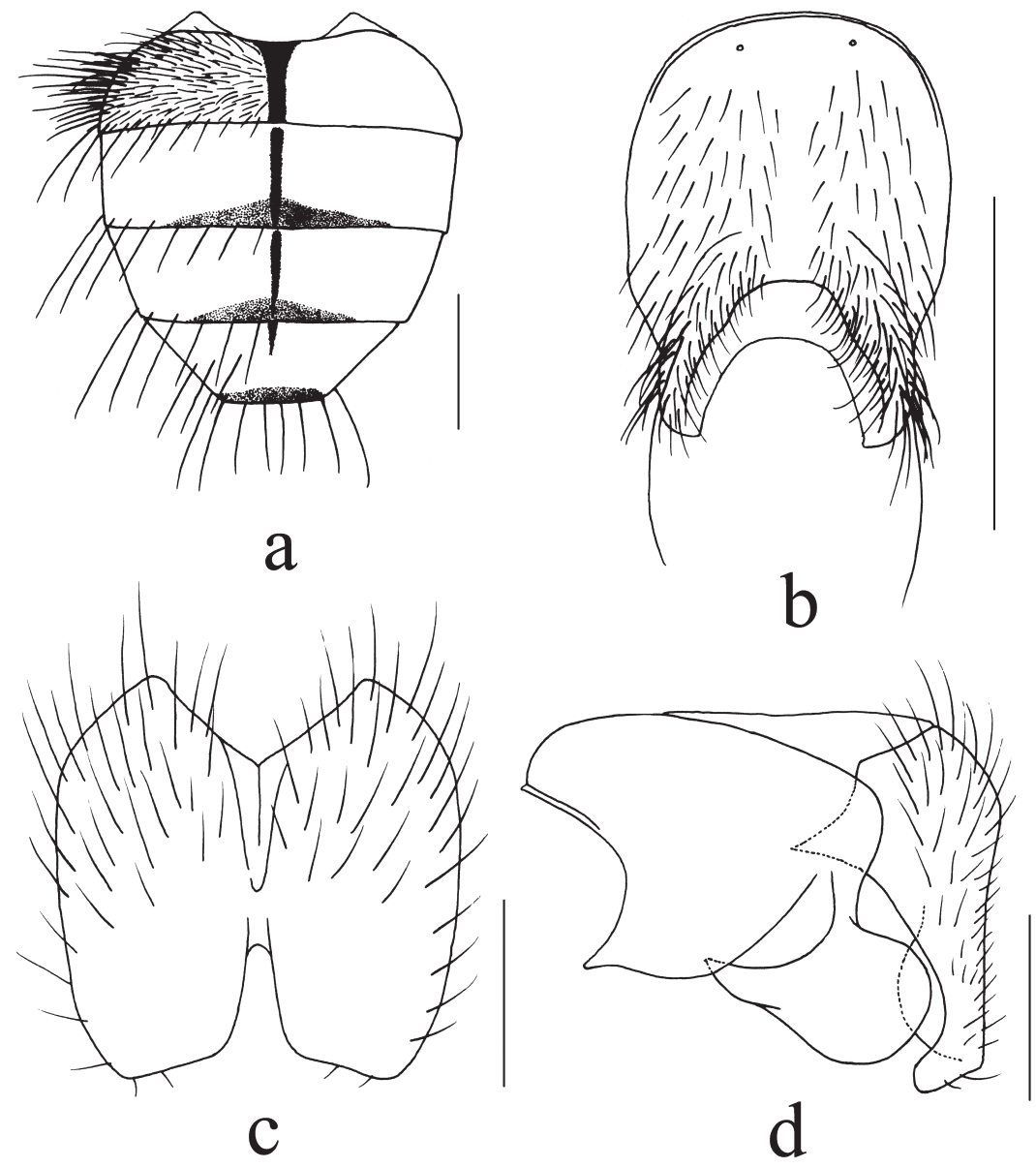

terior view (it is without hairs in posterior view in P. zhangxianggi).

\subsection{Phaonia zhangxianggi Xue \& Yu, sp. n.} (Fig. 3)

Type material. Holotype. Male, China: Moirigkawagarbo (type locality), Yunnan Province $\left(27.02^{\circ} \mathrm{N}, 99.26^{\circ} \mathrm{E}\right.$; altitude 2,900 m), 31.V.2013, Xiang Zhang leg. Paratype. 1 male, same data as holotype.

Description. Male. Body length 6.6-6.8 mm. Head. Eye covered with long brownish ciliae, majority of ciliae at least 3.0 times as long as anterior ocellus width, frons narrower than anterior ocellus width, fronto-orbital plate adjoining, fr 11 to 13 pairs, 5 pairs of $f r$ short and small in upper half, about half of eye ciliae in length, without orbital seta, fronto-orbital plate and parafacial with

grey brownish pruinosity; parafacial without dark patch at the level of pedicel, slightly narrower than antenna width; antenna black, postpedicel 3 times as long as wide, arista long plumose, the longest hair about 1.4 times as long as antenna basal diameter, lunule brownish, epistoma situated front of frontal angle in profile, margin of gena with 2 rows of subvibrissal setulae, gena one third of eye in height, with dark grey pruinosity, occiput dorsally with hairs, gena and postgena entirely black haired; prementum covered with pruinosity, 3.5 times as long as high, palpus thin and long, 1.2 times as long as prementum, all dark brown.

Thorax. Black in ground color, slightly light, sparsely covered with grey pruinosity, scutum with 4 black vittae; inner vittae on scutum not reaching to scutoscutellar suture, acr $0+1, d c$ $2+4$, ial $0+2$, pra about twice as long as posterior notopleural seta, notopleuron with hairs, lateral 
and ventral scutellum bare, basisternum of prosternum, anepimeron, meron and katepimeron all bare, anterior spiracle brownish, posterior spiracle brown, katepisternum setae $1+2$.

Wings. Hyaline, basal part of wing (including veins) yellow, other areas complete brownish, basicosta yellow, costal spine short and small, subequal in length to diameter of $\mathrm{C}$, veins bare, except for $\mathrm{C}$ with hairs, $\mathrm{R}_{4+5}$ and $\mathrm{M}$ straight; calypter and haltere brownish yellow.

Legs. Coxae, trochanter, femora and tibiae yellow, front of fore tibia black, tarsi black; fore tibia $a v 2$, mid femur without distinct $a v$, sub-apical $a 1,4$ to $5 p v$ on basal half, apical $p d 3$, mid tibia $p 3, p v 2$; hind femur with a row of $a v$, longer and stronger apically, without $p v$, hind tibia $a v 3$ to $4, a d 2, p d 1$, a short and small $p d$ sub-basally, without apical $p v$; all tarsi longer than tibiae, hind leg claws and pulvilli short and small, fore leg and mid leg claws and pulvilli subequal in length to fifth tarsomere.

Abdomen. Black in ground color, roundish in dorsal view, covered with slate-grey, slightly shiny pruinosity, each tergite with medium narrow black vitta, posterior margin of tergite 3 to 5 without medium pruinosity, tergite 3 to 5 black, without patch, tergite 4 to 5 posterior marginal seta row complete, sternite 1 bare, sternite 2 to 4 nearly square-shaped, margin of distal part with a pair of strong and thin setae, sternite 5 lateral lobe with a pair of strong setae, margin of cerci apically slightly flattened, inner process proclinate exsert.

Female. Unknown.

Etymology. The species is named after the collector.

Remarks. The new species belongs to the Phaonia acerba group. It resembles P. gaoligongshanensis Xue \& Yu, sp. n., but differs from it in the male characters as shown in the key below. In addition, in Phaonia zhangxianggi Xue \& Yu, sp. n.: palpus dark-brown (palpus black except yellow on basal half, dark-brown on distal half in $P$. gaoligongshanensis), mid tibia with $3 p v$ (mid tibia with $2 p v$ in $P$. gaoligongshanensis), hind tibia with $5 a v$ (hind tibia with 3-4av in P. gaoligongshanensis).

\section{Key to the Palaearctic and Oriental males of the Phaonia acerba group}

1. Post $d c 4$ (P. acerba subgroup) 2

- Post $d c 3$ (P. trisetiacerba subgroup) 7

2. Parafacial with one dark patch at the level of pedicel; gena without subvibrissal setula; $f r$ 3-4 pairs

P. acerba Stein, 1918

- Parafacial without dark patch at the level of pedicel; gena with subvibrissal setula; $f r$ at least 7-8 pairs

3. Basicostablack

- Basicosta yellow

4. Upper parafac yellow, the longest arista hair twice as long as antenna width, trochanters of legs black, hind femur av row complete

P. succinctiantenna Feng \& Ma, 2002

- Upper parafacial without white patch, antenna completely black, the longest arista hair at most 1.5 times as long as antenna width, trochanters of legs yellow, hind femur without $a v$ row on basal half

P. kangdingensis Ma \& Feng, 1985

5. Fr in the lower half of frons, gena one fifth of eye in height, front of fore tibia yellow, mid tibia with $4 p$

\section{P. gaoligongshanensis Xue \& Yu, sp.n.}

- Fr row complete, gena one third of eye in height, front of fore tibia black, mid tibia with $3 p$

6

6. Eye covered with long and thick hairs, majority of ciliae at least 3 times as long as anterior ocellus width, frons narrower than anterior ocellus width, frontal vitta disappearing in middle half, mid tibia with $2 p v$, hind tibia subbasal with an additional $p d$, abdomen oviform in dorsal view, sternite 5 lateral lobe thin and long P.zhangxianggiXue \& Yu, sp.n.

- Eye sparsely covered with short ciliae, the longest ciliae at most 1.5 times as long as anterior ocellus width, frons 1.5 times as wide as anterior ocellus, frontal vitta complete, at least half of fronto-orbital plate in width, mid tibia without $p v$, hind tibia subbasal without $p d$, abdomen inversely triangled in dorsal view, sternite 5 lateral lobe short and wide P. hongkuii Xue \& Yu, sp.n. 
7. Antenna dark yellow; notopleuron entirely covered with black hairs; katepimeron bare, basicosta black brown

P. trisetiacerba Feng \& Ma, 2002

- Antenna black; notopleuron only partially covered with black hairs; katepimeron with black hairs, basicosta black

P. subtrisetiacerba Feng \& Ma, 2002

Acknowledgements. This work was supported by the National Nature Science Foundation of China (No.31172139, No. 30870330), and the Foundation of Experimental Centre of Shenyang Normal University (SYZX200902). Special thanks are due to Feng Y. Tong, Xiang Zhang and Kui H. Sun, who collected the specimens.

\section{References}

Fan, Z. D. 1992: Key to the common flies of China, second edition. - Science Press, Beijing. 992 pp. [In Chinese.]

Hennig, W. 1963: Muscidae. — In: Linder, E. (ed.), Die fliegen der palaearktischen Region 63b: 772-899. Stuttgart. Schweizerbart. 1110 pp.

McAlpine, J. F. 1981: Morphology and terminology adults. - In: McAlpine, J. F., Peterson, B. V., Shewell, G. E., Teskey, H. J., Vockeroth, J. R. \& Wood, D. M. (eds.), Manual of Nearctic Diptera. Volume 1: 9-63. Research, Agriculture Canada Monograph 27. Hull. Canadian Government Publishing Center. 674 pp.

Ringdahl, O. 1949: Forsök till artgruppering inem släktene Phaonia R.-D. och Helina R.-D. (fam. Muscidae). Entomologisk Tidskrift. 70: 136-146. [In Swedish]

Robineau-Desvoidy, A. J. B. 1830: Essai sur les Myodaires. - Memories presentes par divers savantsa l'Academie des sciences de l'Institut de France 2: 1-813.

Shinonaga, S. 2003: A Monograph of the Muscidae of Japan. - University Press, Tunghai. 347 pp.

Stein, P. 1918: Zur weitern Kenntnis aussereuropaeeischer Anthomyiden. - Annales Historico - Naturales Musei Nationalis Hungarici Vienna, 16: 147-244.

Ma, Z. Y. \& Feng, Y. 1985: Six new species of Phaonia from Mounts erlang of Sichuan Province, China. Yaan Science and Technology Press, Yaan. 2: 26-31. [In Chinese.]

Ma, Z. Y. \& Xue, W. Q. \& Feng, Y. 2002: Fauna Sinica Insecta, Volume 26. Diptera: Muscidae II, Phaoniinae I. - Science Press, Beijing. 421 pp. [In Chinese.]

Xue, W. Q. 1996: Muscidae. - In: Xue, W. Q. \& Chao C. M. (eds.), Flies of China, Volume 1: 1166-1298. Liaoning Science and Technology Press, Shenyang, 1365 pp. [In Chinese.]

Zinovjev, A. G. 1981: On the classification of Palaearctic species of the genus Phaonia R-D (Diptera: Muscidae). - Entomologicheskoe Obozrenie. 60(3): 686698. 